\title{
Exact BER Analysis of an Arbitrary Square/ Rectangular QAM for MRC Diversity with ICE in Nonidentical Rayleigh Fading Channels
}

\author{
Laleh Najafizadeh \\ School of Electrical and Computer Engineering \\ Georgia Institute of Tech., Atlanta, Georgia 30332 \\ Email: laleh@ece.gatech.edu
}

\author{
Chintha Tellambura \\ Department of Electrical and Computer Engineering \\ University of Alberta, Canada T6G 2V4 \\ Email: chintha@ece.ualberta.ca
}

\begin{abstract}
In this paper, we derive a general expression for the bit error rate (BER) performance of an arbitrary square/rectangular Gray-coded quadratic amplitude modulation (QAM) scheme. This general formula requires a number of conditional probabilities which we derive in closed-form for independent and nonidentically distributed (i.n.d.) Rayleigh fading channels, when maximum ratio combining (MRC) with imperfect channel estimation (ICE) is employed. Our general formula determines the exact BER of any arbitrary square/rectangular QAM MRC receiver with ICE in i.n.d. Rayleigh fading channels, avoiding time consuming simulations. In addition, Monte Carlo simulations and analytical results are in excellent agreement.
\end{abstract}

\section{INTRODUCTION}

Quadrature amplitude modulation (QAM), a highly spectral efficient modulation scheme, is widely used in wireless communication systems [1]. The bit error rate (BER) performance of $M$-ary QAM has been investigated by several authors. The exact BER for 16-QAM and 64-QAM in an additive white Gaussian noise (AWGN) channel is derived in [2]. Approximate expressions for the BER of an arbitrary $M$ ary square QAM using signal space concepts and recursive algorithms are given in [3] and [4], respectively. Cho and Yoon [5] derive a general expression for the BER of an arbitrary square/rectangular QAM in AWGN. In all these papers, the analysis assumes a perfectly-known channel at the receiver.

For imperfect channel estimation (ICE) less work is available in the literature. Tang et al. [6] derive an approximate expression for the BER of 16-QAM and 64-QAM with ICE over Rayleigh fading channels. In [7], a probability density function (pdf) is introduced to determine the BER of maximum ratio combining (MRC) QAM with channel estimation error. However, the closed-form integrals are obtained only for 16 QAM constellation with 1 and 2 diversity branches for Rayleigh fading and for 1 branch for Rician fading. Cao and Beaulieu [8], [9] provide exact BER expressions for a 16QAM MRC receiver in Rayleigh and Rician fading channels with any number of diversity branches. Their results are only valid for independent and identically distributed (i.i.d.) fading channels. Furthermore, the results in [6]-[9] are presented only for a specific square QAM constellation.
In this paper, we present a general expression for the BER of an arbitrary square/ rectangular QAM with MRC and channel estimation error. First, by analyzing the BER performance of 16-QAM, we observe a regular pattern for the bit error probability of the $k$ th in-phase bit. From this pattern, we develop a general expression for the BER performance of an arbitrary $M$-ary square QAM receiver. This general expression is then extended to an arbitrary $I \times J$-ary rectangular QAM.

The general expressions obtained in this paper require a number of conditional probabilities, based on the channel statistics and the decision variable. For MRC with ICE in i.i.d. fading channels, our several conditional probabilities reduce to the formula provided in [10, Appendix B]. A special case (16-QAM) is considered in [9]. For MRC with ICE in independent and nonidentically distributed (i.n.d.) Rayleigh fading channels, no BER expression is available in the literature. Therefore, we derive an exact and general BER for any arbitrary square/rectangular QAM constellation with MRC and ICE in i.n.d. Rayleigh fading channels.

\section{SySTEM MODEL}

The system model used here is identical to that considered in [7]. MRC is used at the receiver to combat the effects of fading. The received signal sample at each receiver antenna is given by

$$
y_{m}=h_{m} x+n_{m}, \quad m=1 \cdots L
$$

where $x=x_{I}+j x_{Q}, j=\sqrt{-1}, x_{I} \in\{ \pm d, \pm 3 d, \ldots,(I-1) d\}$, $x_{Q} \in\{ \pm d, \pm 3 d, \ldots,(J-1) d\}$, is the data symbol chosen from a $I \times J$ rectangular QAM constellation, $L$ is the number of diversity branches and $h_{m}$ and $n_{m}$ denote the channel attenuation and additive white Gaussian noise of the $m$-th branch, respectively. The channel $h_{m}$ can be estimated by transmitting pilot symbols [11]. At the receiver, the channel estimator outputs

$$
g_{m}=\alpha h_{m}+s_{m}, \quad m=1 \cdots L,
$$

where $\alpha$ is a complex number representing the bias in the estimate and $s_{m}$ is a zero-mean complex Gaussian random 
variable $(\mathrm{RV}){ }^{1}$ with variance $\sigma_{S}^{2}$ representing the random noise in the estimate. Furthermore, we assume that $s_{m}$ and $h_{m}$ are uncorrelated.

With MRC the receiver decision variable is given by [7]

$$
z_{d}=\frac{\sum_{m=1}^{L} y_{m} g_{m}^{*}}{\sum_{m=1}^{L}\left|g_{m}\right|^{2}} .
$$

Since in 2-D modulation schemes the inphase (I) and quadrature $(\mathrm{Q})$ can be demodulated separately, we need the real and imaginary components of $z_{d}$ :

$$
z_{d I}=\operatorname{Re}\left(z_{d}\right)=\frac{\sum_{m=1}^{L} y_{m} g_{m}^{*}+y_{m}^{*} g_{m}}{2 \sum_{m=1}^{L}\left|g_{m}\right|^{2}}
$$

and

$$
z_{d Q}=\operatorname{Im}\left(z_{d}\right)=\frac{\sum_{m=1}^{L} y_{m} g_{m}^{*}-y_{m}^{*} g_{m}}{2 j \sum_{m=1}^{L}\left|g_{m}\right|^{2}} .
$$

Define $w_{m}=\left(g_{m}, y_{m}\right)$. We now compute the covariance matrix of $w_{m}$ which will be used in the following sections. In Rayleigh fading, the covariance matrix of $w_{m}$ is given by

$$
C_{w_{m}}=E\left[w_{m} w_{m}^{\dagger}\right]=\left(\begin{array}{ll}
\sigma_{g g_{m}}^{2} & \sigma_{g y_{m}}^{2} \\
\sigma_{y g_{m}}^{2} & \sigma_{y y_{m}}^{2}
\end{array}\right)
$$

where $\sigma_{g g_{m}}^{2}, \sigma_{y y_{m}}^{2}$ and $\sigma_{g y_{m}}$ denote the variances of $y_{m}, g_{m}$ and the covariance of $g_{m}$ and $y_{m}$, respectively and are defined as

$$
\begin{gathered}
\sigma_{g g_{m}}^{2}=E\left(g_{m} g_{m}^{*}\right)=|\alpha|^{2} E\left(\left|h_{m}\right|^{2}\right)+E\left(\left|s_{m}\right|^{2}\right) \\
\sigma_{y y_{m}}^{2}=E\left(y_{m} y_{m}^{*}\right)=|x|^{2} E\left(\left|h_{m}\right|^{2}\right)+E\left(\left|n_{m}\right|^{2}\right) \\
\sigma_{g y_{m}}^{2}=E\left(g_{m} y_{m}^{*}\right)=\alpha x^{*} E\left(\left|h_{m}\right|^{2}\right) .
\end{gathered}
$$

Note that the subscript $\dagger$ represents conjugate transpose.

\section{Signal Model}

We consider $I \times J$ rectangular QAM consisting of two independent one-dimensional amplitude modulation (PAM) signals. In the special case when $I=J$, the constellation represents an $M$-ary square QAM, where $M=I \times I$. We also assume that all the transmit symbols are equally likely.

In a typical QAM modulator, the data stream is divided into the I and Q bit streams, each encoded onto a separate axis using identical Gray coding mapping blocks [1]. The modulated signal is represented by

$$
s(t)=A_{I} \cos 2 \pi f_{c} t-A_{J} \sin 2 \pi f_{c} t, \quad 0 \leq t \leq T
$$

${ }^{1}$ We say $x=x_{I}+j x_{Q}$ is a complex Gaussian RV denoted by $x \backsim C N\left(\mu, \sigma^{2}\right)$, if $x_{I}$ and $x_{Q}$ are real Gaussian RVs with mean $\mu_{x}$ and $\mu_{y}$ and variance $\sigma_{x}^{2}$ and $\sigma_{y}^{2}$, respectively and $\mu=\mu_{x}+j \mu_{y}, \sigma^{2}=\sigma_{x}^{2}+\sigma_{y}^{2}$. where $f_{c}$ is the carrier frequency, $T$ is the symbol time duration and $A_{I}$ and $A_{J}$ are the amplitudes of the in-phase and quadrature components selected independently over the set of $\{ \pm d, \pm 3 d, \ldots,(I-1) d\}$ and $\{ \pm d, \pm 3 d, \ldots,(J-1) d\}$, respectively. Note that $2 d$ is the Euclidean distance between two adjacent signal points. Denoting $E_{b}$ as the bit energy, $d$ can be written in terms of $E_{b}, I$ and $J$ as [5]

$$
d=\sqrt{\frac{3 E_{b} \log _{2}(I \times J)}{I^{2}+J^{2}-2}} .
$$

For the case of $M$-ary square QAM, (8) becomes

$$
d=\sqrt{\frac{3 E_{b} \log _{2} M}{2(M-1)}} .
$$

At the receiver, the complex symbols are divided into I and Q components and each component is demodulated independently against their respective decision boundaries.

We next derive a general BER expression for $M$-ary square and $I \times J$ rectangular QAM using conditional probabilities.

\section{GENERAL BER EXPRESSION FOR $M$-ARY SQUARE QAM}

A general expression for the BER of an arbitrary square/rectangular QAM for AWGN channels is derived in [5]. However, it depends on complementary error function and is only valid for AWGN channels. Here, we derive a general expression for the BER of M-ary QAM which is valid not only in AWGN but also in any fading channel with/without diversity and with perfect/imperfect channel estimation.

Due to symmetry, the average BER of an $M$-ary square QAM is equal to the BER of either the I or the Q components. We assume that in QAM, the most significant bit is the inphase bit. The BER of an $M$-ary square QAM is given by

$$
P_{b}=\frac{1}{\log _{2} \sqrt{M}} \sum_{n=0}^{\log _{2} \sqrt{M}-1} P_{I}\left(\log _{2} M-2 n\right),
$$

where $P_{I}\left(\log _{2} M-2 n\right)$ denotes the bit error probability of the $\left(\log _{2} M-2 n\right)$-th bit in the in-phase components. Therefore, in order to obtain the average BER of an $M$-ary square QAM one needs to determine $P_{I}\left(\log _{2} M-2 n\right)$. In this section, we first determine $P_{I}\left(\log _{2} M-2 n\right)$ for the two in-phase bits of a 16-QAM constellation. Using this example, we then obtain a general expression for the bit error probability of the $\left(\log _{2} M-\right.$ $2 n$ )-th in-phase bit of an arbitrary $M$-ary square QAM. Using (10) the BER of an $M$-ary square QAM is determined.

\section{A. BER of 16-QAM}

Figure 1 shows the constellation, Gray-coded bit mapping and the decision boundaries for a square 16-QAM. It can be seen that the fourth ${ }^{2}$ and the second bits are the in-phase bits while the third and the first bits are the quadrature bits. Due to symmetry, we only define the bit error probability of the in-phase bits.

\footnotetext{
${ }^{2}$ In this paper, the least significant bit is referred to as the first bit and the
} most significant bit is referred to as the $\left(\log _{2} M\right)$-th bit. 


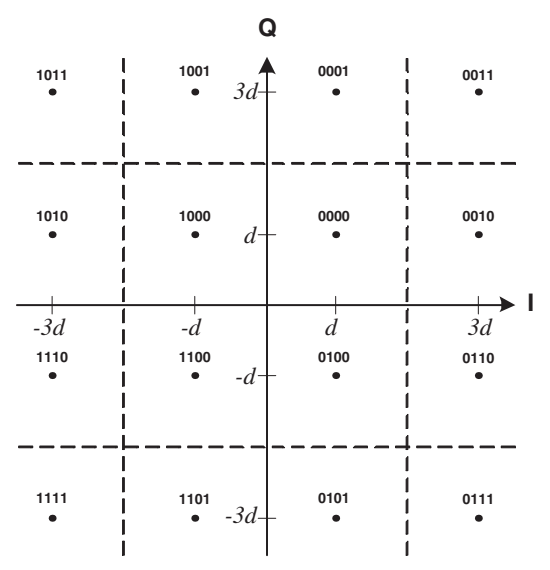

Fig. 1. Square 16-QAM constellation with Gray encoding.

The Q-axis is the decision boundary for the fourth bit. Because of the symmetry between the right and left sides of the plane, the probability that the fourth bit is in error is [2]

$$
\begin{aligned}
P_{I}(4)= & \frac{1}{2}\left[\operatorname{Pr}\left(\left(z_{d I}<\left.0\right|_{\operatorname{Re}(x)=d}\right)+\operatorname{Pr}\left(z_{d I}<\left.0\right|_{\operatorname{Re}(x)=3 d}\right)\right]\right. \\
= & \frac{1}{4}\left[\operatorname{Pr}\left(z_{d I}<\left.0\right|_{x=d+j d}\right)+\operatorname{Pr}\left(z_{d I}<\left.0\right|_{x=d+j 3 d}\right)\right. \\
& \left.+\operatorname{Pr}\left(z_{d I}<\left.0\right|_{x=3 d+j d}\right)+\operatorname{Pr}\left(z_{d I}<\left.0\right|_{x=3 d+j 3 d}\right)\right] .
\end{aligned}
$$

For the second bit, the decision boundaries are $2 d$ and $-2 d$. If the second bit is a "0", the error occurs when the real part of decision variable, $z_{d}$, is out of the region $(-2 d, 2 d)$ and if the second bit is " 1 ", an error occurs if the real part of $z_{d}$ falls in $(-2 d, 2 d)$. Therefore, the error probability of the second bit is obtained as [2]

$$
\begin{aligned}
P_{I}(2)= & \frac{1}{2}\left[\operatorname{Pr}\left(\left|z_{d I}\right|>\left.2 d\right|_{\operatorname{Re}(x)=d}\right)+\operatorname{Pr}\left(\left|z_{d I}\right|<\left.2 d\right|_{\operatorname{Re}(x)=3 d}\right)\right] \\
= & \frac{1}{4}\left[1-\operatorname{Pr}\left(z_{d I}<\left.2 d\right|_{x=d+j d}\right)-\operatorname{Pr}\left(z_{d I}<\left.2 d\right|_{x=d+j 3 d}\right)\right. \\
& +1+\operatorname{Pr}\left(z_{d I}<-\left.2 d\right|_{x=d+j d}\right)+\operatorname{Pr}\left(z_{d I}<-\left.2 d\right|_{x=d+j 3 d}\right) \\
& +\operatorname{Pr}\left(z_{d I}<\left.2 d\right|_{x=3 d+j d}\right)+\operatorname{Pr}\left(\left.z_{d I}\right|_{x=3 d+j 3 d}\right) \\
& \left.-\operatorname{Pr}\left(z_{d I}<-\left.2 d\right|_{x=3 d+j d}\right)-\operatorname{Pr}\left(z_{d I}<-\left.2 d\right|_{x=3 d+j 3 d}\right)\right] .
\end{aligned}
$$

Substituting (11) and (12) in (10) gives BER for 16-QAM.

\section{B. General BER Expression for an M-ary Square $Q A M$}

From (11) and (12) a regular pattern can be found for the bit error probability of the $\left(\log _{2} M-2 n, n \in\left\{0,1, \cdots, \log _{2} \sqrt{M}-\right.\right.$ 1\})-th bit as

$$
\begin{aligned}
& P_{I}\left(\log _{2} M-2 n\right)= \\
& \begin{cases}\frac{4}{M}\left[\sum_{\substack{k=1 \\
k \text { odd }}}^{\sqrt{M}-1} A_{k, 0}\right] & (n=0) \\
\frac{4}{M}\left[\frac{M}{8}+\sum_{i=0}^{2^{n-1}} \sum_{\substack{k=F_{1}(i, n) \\
k \text { odd }}}^{F_{2}(i, n)}(-1)^{i} A_{k, n}\right] & (n \neq 0)\end{cases}
\end{aligned}
$$

where $F_{1}(i, n)=\max \left[\frac{\sqrt{M}}{2^{n}}(2 i-1)+1, \quad 1\right], \quad F_{2}(i, n)=$ $\min \left[\frac{\sqrt{M}}{2^{n}}(2 i+1)-1, \sqrt{M}-1\right]$ and

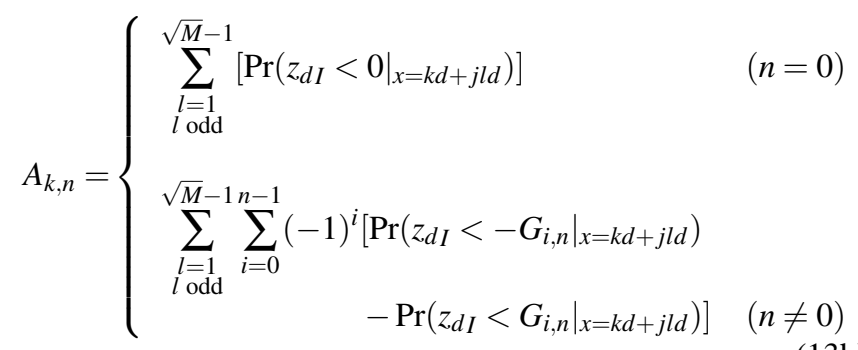

where $G_{i, n}=\frac{\sqrt{M}(2 i+1)}{2^{n}} d$.

If the conditional probabilities are known, then the above expressions for $P_{I}\left(\log _{2} M-2 n\right)$ can be used in (10) to obtain the BER of an $M$-ary square QAM.

\section{GENERAL BER EXPRESSION FOR $I \times J$ RECTANGULAR QAM}

In this section we extend the analysis presented in the previous section to an arbitrary $I \times J$ rectangular QAM.

If we assume that the most significant bit is the in-phase bit, then the BER of an $I \times J$ rectangular QAM is given by

$$
\begin{aligned}
P_{b}=\frac{1}{\log _{2}(I \times J)} \times & {\left[\sum_{n=0}^{\log _{2} I-1} P_{I}\left(\log _{2}(I \times J)-2 n\right)\right.} \\
& \left.+\sum_{n=0}^{\log _{2} J-1} P_{Q}\left(\log _{2}(I \times J)-(2 n+1)\right)\right],
\end{aligned}
$$

where $P_{I}\left(\log _{2}(I \times J)-2 n\right)$ and $P_{Q}\left(\log _{2}(I \times J)-(2 n+1)\right)$ denote the bit error probabilities of the $\left(\log _{2}(I \times J)-2 n\right)$ th in-phase bit and $\left(\log _{2}(I \times J)-(2 n+1)\right)$-th quadrature bit, respectively. The general expression for the BER of the inphase bits is given in Section IV. Note that for the case of $I \times J$ rectangular QAM, $M$ and $\sqrt{M}$ in (13) should be replaced by $I \times J$ and $I$, respectively.

For the quadrature bits, the imaginary part of the decision variable should be considered. The regular pattern that can be found for the bit error probability of the $\left(\log _{2} I \times J-(2 n+1)\right)$ th bit is

$$
\begin{aligned}
& P_{Q}\left(\log _{2}(I \times J)-(2 n+1)\right)= \\
& \begin{cases}\frac{4}{I \times J}\left[\sum_{\substack{k=1 \\
k \text { odd }}}^{J-1} B_{k, 0}\right] & (n=0) \\
\frac{4}{I \times J}\left[\frac{I \times J}{8}+\sum_{i=0}^{2^{n-1}} \sum_{\substack{k=E_{1}(i, n) \\
k \text { odd }}}^{E_{2}(i, n)}(-1)^{i} B_{k, n}\right] & (n \neq 0)\end{cases}
\end{aligned}
$$


where $E_{1}(i, n)=\max \left[\frac{J}{2^{n}}(2 i-1)+1,1\right], E_{2}(i, n)=\min \left[\frac{J}{2^{n}}(2 i+\right.$ 1) $-1, J-1]$ and

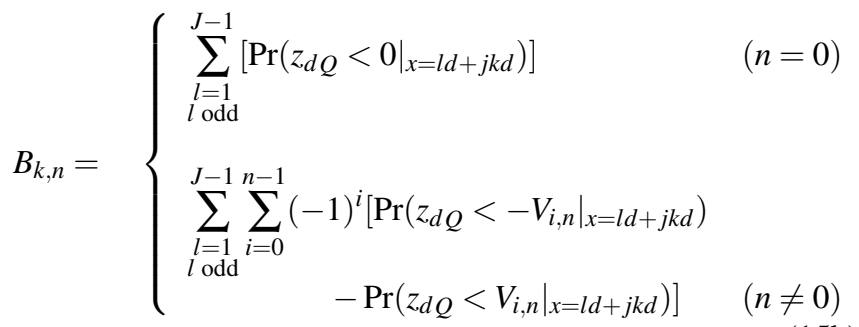

where $V_{i, l}=\frac{J(2 i+1)}{2^{l}} d$.

Once $P_{I}\left(\log _{2}(I \times J)-2 n\right)$ and $P_{Q}\left(\log _{2}(I \times J)-(2 n+1)\right)$ are defined from (13) and (15), the BER of an $I \times J$ rectangular QAM follows from (14).

The above analysis shows that the BER of an arbitrary square/ rectangular QAM consists of the summation of the probability terms in the form of $\operatorname{Pr}\left(z_{d I}<\left.T\right|_{x=a d+j b d}\right)$ and $\operatorname{Pr}\left(z_{d Q}<\left.T\right|_{x=a d+j b d}\right)$ where $a \in\{1,3, \ldots,(I-1)\}, b \in$ $\{1,3, \ldots,(J-1)\}$ and $T$ is an arbitrary number. We next determine the expression for these probabilities when MRC in i.n.d. Rayleigh fading channels with ICE is used at the receiver.

\section{EXACT BER EXPRESSION FOR AN ARBITRARY SQUARE/RECTANGULAR QAM IN RAYLEIGH FADING CHANNELS}

To find a closed-form expression for the conditional probabilities in (13) and (15) for MRC with ICE, we consider two separate cases.

\section{A. i.i.d. Fading Channels}

In i.i.d. fading channels, $h_{m}$ and $g_{m}$ in (3) are i.i.d. Gaussian RVs. With MRC in i.i.d. Rayleigh or Rician fading channels the conditional probabilities in (13) and (15) are reduced to a closed-form expression given in [10, Eq.(B-21)]. Our general expression then can be used for the derivation of the BER of any square/rectangular QAM scheme with MRC and ICE in i.i.d. Rayleigh/ Rician fading channels. A special case of 16-QAM is considered in [9].

\section{B. i.n.d. Fading Channels}

We now find a closed-form expression for the conditional probabilities $\operatorname{Pr}\left(z_{d I}<\left.T\right|_{x=a d+j b d}\right)$ and $\operatorname{Pr}\left(z_{d Q}<\left.T\right|_{x=a d+j b d}\right)$ in equations (13) and (15) for the case of i.n.d. Rayleigh fading channels with ICE.

Using (4a), the $\operatorname{Pr}\left(z_{d I}<\left.T\right|_{x=a d+j b d}\right)$ can be rewritten as

$\operatorname{Pr}\left(z_{d I}<\left.T\right|_{x=a d+j b d}\right)=\operatorname{Pr}\left(\frac{\sum_{m=1}^{L} y_{m} g_{m}^{*}+y_{m}^{*} g_{m}}{2 \sum_{m=1}^{L}\left|g_{m}\right|^{2}}<\left.T\right|_{x=a d+j b d}\right)$ $=\operatorname{Pr}\left(D_{I}<\left.0\right|_{x=a d+j b d}\right)$

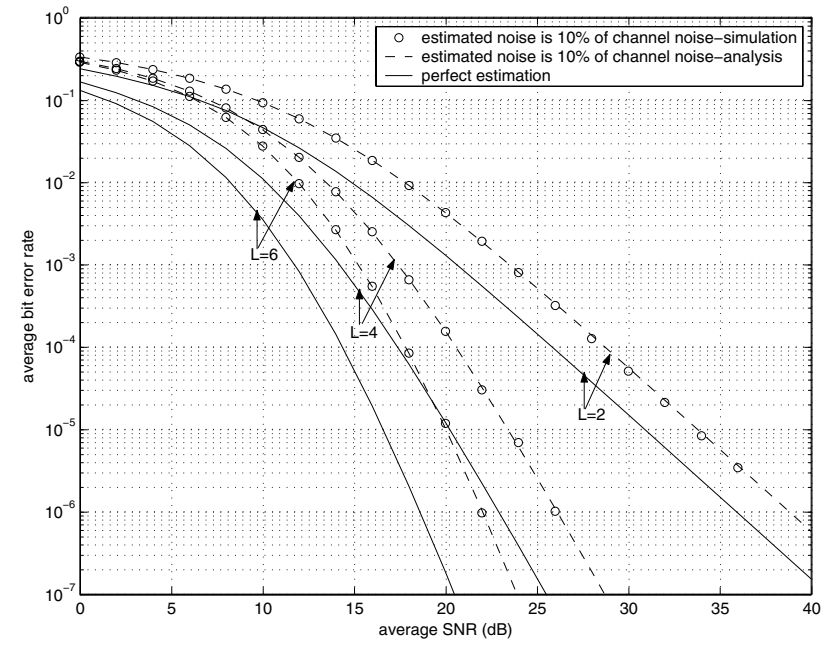

Fig. 2. Plot of BER of an 16-QAM with ICE case 1 and $\beta=0.5$ for three different diversity branches.

where

$$
D_{I}=\sum_{m=1}^{L}\left(-2 T\left|g_{m}\right|^{2}+y_{m} g_{m}^{*}+y_{m}^{*} g_{m}\right)=\sum_{m=1}^{L} d_{I_{m}}
$$

is a special case of the general quadratic form. Note that $d_{I_{m}}$ in (17) can be written as

$$
d_{I_{m}}=w_{m} H_{I} w_{m}^{\dagger}
$$

where $w_{m}=\left(\begin{array}{ll}g_{m} & y_{m}\end{array}\right)$ and $H_{I}=\left(\begin{array}{cc}-2 T & 1 \\ 1 & 0\end{array}\right)$.

Similarly, using (4b), $\operatorname{Pr}\left(z_{d Q}<\left.T\right|_{x=a d+j b d}\right)$ can be expressed as

$$
\operatorname{Pr}\left(z_{d Q}<\left.T\right|_{x=a d+j b d}\right)=\operatorname{Pr}\left(D_{Q}<\left.0\right|_{x_{Q}=a d+j b d}\right)
$$

where

$$
D_{Q}=\sum_{m=1}^{L} d_{Q_{m}}=\sum_{m=1}^{L} w_{m} H_{Q} w_{m}^{\dagger}
$$

and

$$
H_{Q}=\left(\begin{array}{cc}
-2 T & \frac{1}{j} \\
\frac{-1}{j} & 0 .
\end{array}\right) .
$$

We use the moment generating function approach to find closed-form expressions for these probabilities. The details can be found in our journal paper. With these closed-form expressions for the conditional probabilities in (13) and (15), the BER of an arbitrary square/rectangular QAM with MRC and ICE in i.n.d. Rayleigh fading follows from (10) and (14).

\section{Simulations}

All the simulations in this section are implemented in Matlab. Two ICE cases are considered as in [7]: case $1, \alpha=1$ and $\sigma_{S}^{2}=0.1 \sigma_{N}^{2}$ which shows that the estimation noise is $10 \%$ of the channel noise; case 2, in addition to the estimation noise, an irreducible error in the estimation of the channel is present, i.e. $\sigma_{S}^{2}=0.0001 \sigma_{h}^{2}+0.1 \sigma_{N}^{2}$. 


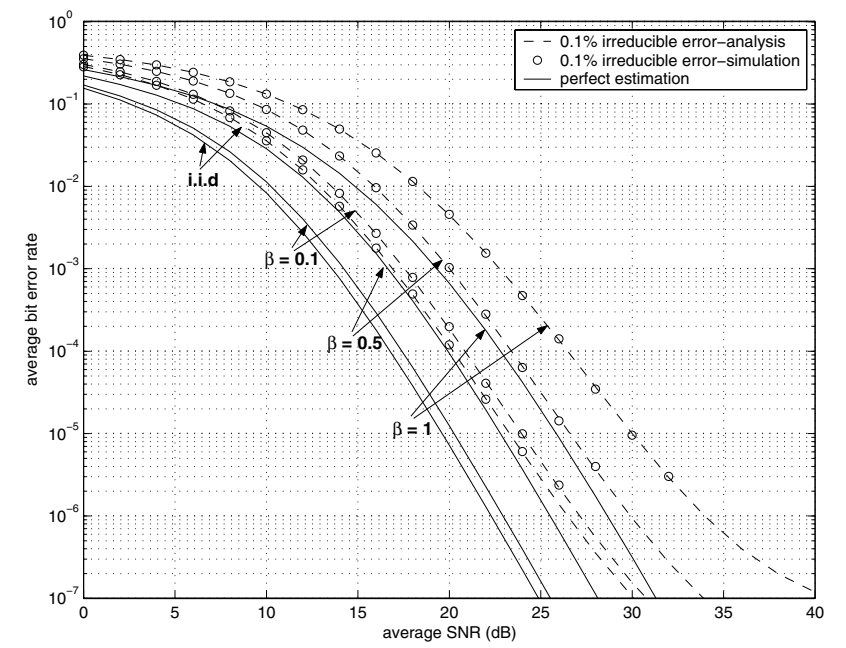

Fig. 3. Plot of BER of an 16-QAM with 4 diversity branches and ICE case 2 for four different channel MIP's.

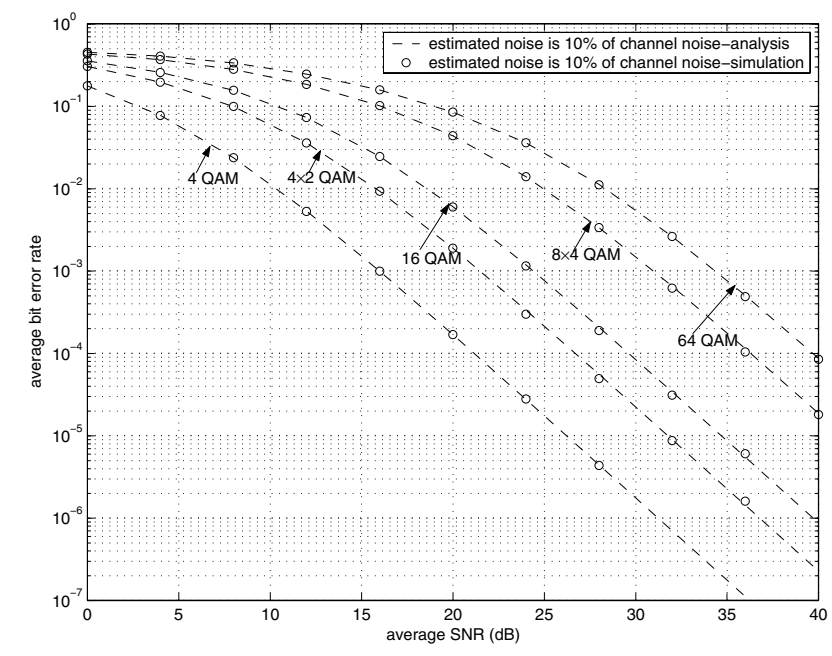

Fig. 4. Plot of BER of several constellations with two diversity branches and ICE case 1 and $\beta=0.1$.

For i.n.d. fading channels, we consider an exponentially multipath intensity profile (MIP) with decay parameter $\beta$, i.e. $\gamma_{l}=\gamma_{1} \exp \{-\beta(l-1)\}, l=1,2, \cdots, L$.

For analytical results, we used our result from Section VI$B$ in (13) and (15) and then in (10) and (14) to obtain the BER performance of MRC QAM with ICE. In Figure 2, the BER performance of MRC 16-QAM for case 1 and for three different diversity branches is presented. The MIP decay parameter, $\beta$, is set to 0.5 . For both perfect and imperfect estimation, the BER performance improves when the number of diversity branches increases. Also for case 1, ICE does not change the slope of the BER curves. Interestingly, as the diversity order increases, the SNR difference between perfect channel estimation and ICE increases. For example, at a BER of $10^{-4}$, the SNR difference between perfect and imperfect estimation for $\mathrm{L}=2,4$ and 6 is $2.89 \mathrm{~dB}, 3.19 \mathrm{~dB}$ and 3.46
$\mathrm{dB}$, respectively. This shows that as the number of diversity branches increases, the degradation of ICE becomes more significant.

Figure 3 shows the BER of a four branch reception 16-QAM for four different channel MIPs for case 2. The BER with perfect estimation for each $\beta$ is also presented for comparison. The i.i.d. channel case has the best BER performance and the BER performance degrades as the decay exponent, $\beta$, increases. For case 2, an error floor occurs at high SNR.

We used (13) and (15) to plot the BER performances of several square and rectangular QAM constellations. We assumed two branch MRC receiver and $\beta=0.1$ and ICE case 1. The analytical results along with Monte Carlo simulations are shown in Figure 4. This figure shows that to transmit an extra bit per dimension, the SNR must be increased by 3-6 $\mathrm{dB}$.

\section{CONCLUSION}

In this paper, we have derived a general expression for the BER of an arbitrary square/rectangular QAM. This requires several conditional probabilities which can be obtained in closed-form. A closed-form expression was given for the BER of square/rectangular MRC QAM with ICE in i.n.d. Rayleigh fading channels. Our results are valid for any number of diversity branches. The validity of our analytical results is confirmed by Monte Carlo simulations. More extensive results and a further discussion can be found in our journal paper.

\section{REFERENCES}

[1] L. Hanzo, W. Webb, and T. Keller, Single- and Multi-carrier Quadrature Amplitude Modulation: Principles and Applications for Personal Communications, WLANs and Broadcasting. Chinchester, UK: John Wiley and Sons, 2002.

[2] M. P. Fitz and J. P. Seymour, "On the Bit Error Probability of QAM Modulation," International Journal of Wireless Information Networks, vol. 1, no. 2, pp. 131-139, 1994.

[3] J. Lu, K. Letaief, J. C.-I. Chung, and M. L. Liou, "M-PSK and MQAM BER Computation using Signal-Space Concepts," IEEE Trans. on Commun., vol. 47, no. 2, pp. 181-184, Feb. 1999.

[4] L. Yang and L. Hanzo, "A Recursive Algorithm for the Error Probability Evaluation of M-QAM," IEEE Communications Letter, vol. 4, pp. 304306, Oct. 2000.

[5] K. Cho and D. Yoon, "On the General BER Expression of One- and Two Dimensional Amplitude Modulations," IEEE Trans. on Commun., vol. 50, no. 7, pp. 1074-1080, July 2002.

[6] X. Tang, M.-S. Alouini, and A. J. Goldsmith, "Effect of Channel Estimation Error on M-QAM BER Performance in Rayleigh Fading," IEEE Trans. on Commun., vol. 47, no. 12, pp. 1856-1864, Dec. 1999.

[7] S. K. Wilson and J. M. Cioffi, "Probability Density Functions for Analyzing Multi-Amplitude Constellations in Rayleigh and Ricean Channels," IEEE Trans. on Commun., vol. 46, no. 3, pp. 380-386, Mar 1999.

[8] L. Cao and N. C. Beaulieu, "Exact Error Rate Analysis of Diversity 16-QAM with Channel Estimation Error," IEEE Trans. on Commun., vol. 52, no. 6, pp. 1019-1029, June 2004.

[9] L. Cao and N. C. Beaulieu, "Closed-Form BER Results for MRC Diversity with Channel Estimation Errors in Ricean Fading Channels," IEEE Trans. on Wirelss Commun., vol. to appear.

[10] J. G. Proakis, Digital Communications, fourth ed. New York: McGrawHill, 2001.

[11] J. K. Cavers, "An Analysis of Pilot-Symbol-Assisted Modulation for Rayliegh Fading Channels," IEEE Trans. Veh. Tech., vol. 40, pp. 1389 1399, Nov. 1991. 